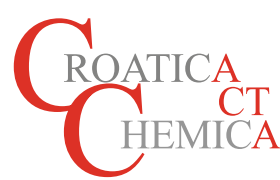

\title{
Nucleofugality of Leaving Groups
}

Mirela Matić, Bernard Denegri, Sandra Jurić, Olga Kronja*

\footnotetext{
University of Zareb, Faculty of Pharmacy and Biochemistry, Ante Kovačića 1, HR-10000 Zagreb, Croatia

* Corresponding author's e-mail address: okronja@pharma.hr
}

RECEIVED: January 11, 2018 * REVISED: April 9, 2018 * ACCEPTED: April 9, 2018

THIS PAPER IS DEDICATED TO PROF. MLADEN ŽINIĆ ON THE OCCASION OF HIS 70 ${ }^{\mathrm{TH}}$ BIRTHDAY

\begin{abstract}
In this short authors' review, a method for determining experimental and calculated nucleofugalities of leaving groups in a given solvents according to LFER equation log $k=s_{f}\left(N_{f}+E_{f}\right)$ is presented. Also, a comprehensive overview of the experimental and calculated nucleofuges specific parameters $\left(N_{f}\right.$ and $\left.s_{f}\right)$ for various negatively charged and neutral leaving groups is shown. Some applications of the above method have been demonstrated: use of the electrofugalitiy and nucleofugality scales to estimate the reactivities of a variety of substrates in various solvents, as well as assessment whether a given substrate is stable in a given solvent for a sufficent amount of time, which may indicate if the substrate can be handled in the solvent of choice during synthetic and other procedures. The method can also be used to establish whether the relative reactivity of leaving groups depends on the electrofuge moiety of the substrate.
\end{abstract}

Keywords: nucleofugality, electrofugality, leaving group, solvolysis.

\section{INTRODUCTION}

$\mathbf{T}$ HE initial step of reactions in which the substrates solvolyze according to the $\mathrm{S}_{\mathrm{N}} 1$ pathway involves the heterolytic cleavage of a carbon-LG (leaving group) bond and formation of a carbocation intermediate (electrofuge) and a free leaving group (nucleofuge) (Scheme 1). ${ }^{[1]}$ The influence of fine structural features of electrofuges on substrate reactivity has thoroughly been investigated back from the mid twentieth century using most of the arsenal<smiles>[X]c1cccc(C(C)c2cccc([Y])c2)c1</smiles>
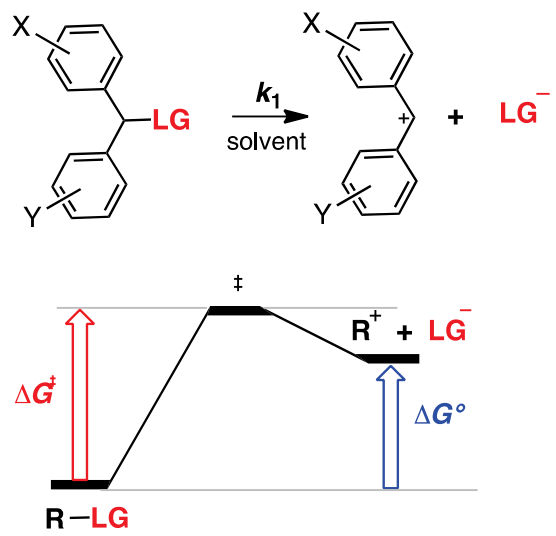

Scheme 1. of physical organic chemistry. Reaction rates of reference and perturbed compounds have been compared, kinetic and equilibrium isotope effects have been measured, correlation analysis, isotope labelling studies as well as product analysis have been carried out, etc. ${ }^{[2]}$ On the other hand, effects of leaving groups have mostly been neglected and the phenomena observed have mainly been attributed to the effect of electrofuges (carbocations) only, as well as to solvation effects.

Reactivities of leaving groups have been associated with their Lewis basicity. ${ }^{[1]}$ According to the general qualitative rule of thumb, a weaker base constitutes a better, more reactive, leaving group, i.e., LGs are arranged in the same order as acidities of their conjugate Brönsted acids. Yet, there are some basic shortcomings of such approach. The Lewis basicity toward a proton may be different than toward a carbocation. Further, the reactivity of LG, nucleofugality, is reflected in activation free energy $\left(\Delta G^{\ddagger}\right)$ and therefore also in the heterolytic rate constant $k_{1}$, hence it constitutes a kinetic term, while the basicity is the thermodynamic term determined with $\Delta G^{\circ}$ (Scheme 1). By predicting relative reactivities of LGs by comparing their Lewis basicities, the impact of an intrinsic barrier is completely neglected since the free energy of activation $\left(\Delta G^{\ddagger}\right)$ is, beside with $\Delta G^{\circ}$, determined also with the intrinsic

(c) $\mathbf{B Y}$ This work is licensed under a Creative Commons Attribution 4.0 International License. 
barrier. ${ }^{[3,4,5]}$ Some examples show that the solvolytic behaviour of substrates with structurally similar LGs are in line with the Hammond postulate ${ }^{[6]}$ and with the BellEvans-Polanyi principle, ${ }^{[7]}$ i.e., as their rates decrease, the endergonicity of the heterolysis step increase. ${ }^{[4,8,9]}$ In such cases, the order of the reactivity of LGs can be related to the order of their basicity. However, for structurally different leaving groups (nucleofuges) correlation between the basicity and reactivity does not necessarily exist. ${ }^{[4,5]}$ Clear evidence that the Lewis basicity is not suitable for determination of the relative reactivity of leaving groups is given in the following examples. Tosylates solvolyze about five orders of magnitude faster than the corresponding chlorides (the solvolysis rate ratio of adamantyl derivatives is $k_{\mathrm{AdOTS}} / k_{\mathrm{AdCl}}=5 \times 10^{5}$ in $80 \%$ aq. ethanol), ${ }^{[10]}$ while hydrochloric acid $\left(\mathrm{p} K_{\mathrm{a}}=-6.3\right.$ in water) ${ }^{[11 a]}$ is a stronger acid than $p$-toluenesulfonic acid $\left(\mathrm{p} K_{\mathrm{a}}=-2.8 \text { in water }\right)^{[11 \mathrm{~b}]}$ Similar discrepancy exists, for example, between the solvolytic reactivity of phenolates and carboxylates. Thus, 4-methoxybenzhydryl 2,4dinitrophenolate ${ }^{[12]}$ solvolyzes about 150 times faster than the corresponding chloroacetate ${ }^{[8]}$ in $80 \%$ aq. ethanol, although chloroacetate $\left(\mathrm{p} K_{\mathrm{a}}=2.87\right.$ for chloroacetic acid) ${ }^{[13 a]}$ is a weaker base than dinitrophenolate ( $\mathrm{p} K_{\mathrm{a}}=4.09$ for 2,4-dinitrophenol). ${ }^{[13 \mathrm{~b}]}$

The first attempt to systematically quantify the reactivity of leaving groups comes from Noyce, who compared reactivities of some leaving groups based on relative reaction rates of 1 -phenylethyl-LG in $80 \%$ aq. ethanol. ${ }^{[14]}$ However, by using a single electrofuge (1phenylethyl) while varying the leaving groups, the rates can reliably be obtained in a relatively narrow range of reactivities at a given temperature. The range can only moderately be extended if the rate constants are extrapolated from those obtained at lower or higher temperatures. Noyce somewhat extended the reactivity range of the leaving groups by including rate constants for substituted 1-phenylethyl derivatives and presuming constant rate ratios.

In collaboration with Mayr's group (LMU, München) we have proposed an approach analogous to that used for establishing the most comprehensive electrophilicity and nucleophilicity scales, in which the contribution of an electrophile and a nucleophile to the combination reaction rate is treated separately according to three parameter LFER equation: $\log k=s(N+E)$. $^{[15]}$ The comprehensive nucleofugality and electrofugality scales have been developed on the basis of solvolysis rates of benzhydryl derivatives. ${ }^{[16]}$ The contributions of nucleofuges and electrofuges to the overall solvolytic reactivity are defined individually, so the heterolysis rate constant of any substrate in a given solvent at $25^{\circ} \mathrm{C}$ can be expressed by the following three-parameter LFER equation:[16]

$$
\log k=s_{f}\left(N_{f}+E_{f}\right)
$$

in which $k$ is the first-order rate constant $\left(\mathrm{s}^{-1}\right)$ at $25^{\circ} \mathrm{C}, s_{\mathrm{f}}$ is the nucleofuge-specific slope parameter, $N_{f}$ is the nucleofugality parameter, and $E_{f}$ is the electrofugality parameter. The $E_{f}$ parameter is set up as a solvent independent variable that refers to the ability of a carbocation to depart from a substrate in the heterolysis reaction $\left(S_{N} 1\right)$. Since the nucleofugality of a leaving group depends not only on the substrate structure but also on the nature of a solvent, the nucleofugality for each leaving group is given in the combination with a solvent. Thus, here the nucleofuge-specific parameters $\left(s_{f}\right.$ and $N_{f}$ ) describe the leaving group ability in a given solvent. Predefined parameters are: $E_{f}=0.00$ for the dianisylcarbenium electrofuge and $s_{f}=1.00$ for the chloride nucleofuge in pure ethanol.[16] According to Equation (1), the nucleofugality $\left(N_{\mathrm{f}}\right)$ of a given leaving group is defined as the negative intercept on the abscissa of the log $k v s$. $E_{\mathrm{f}}$ correlation line. The absolute rates of heterolysis reactions $\left(S_{N} 1\right)$ for various combinations of electrofuge-nucleofuge can be estimated according to Equation (1).

In this short authors' review, a brief presentation of experimental procedures for collecting rate constants used for establishing the nucleofugality/electrofugality scales and computational methods for calculation of the nucleofuge-specific parameters is provided. Further, a comprehensive overview of the reactivity of numerous leaving groups is given by presenting their nucleofugespecific parameters $\left(N_{f}\right.$ and $\left.s_{f}\right)$ in various solvents, along with some possible applications of these data in everyday laboratory practice.

\section{EXPERIMENTAL NUCLEOFUGE- SPECIFIC PARAMETERS}

The nucleofugality scale for a wide range of leaving groups was developed by using kinetic data for benzhydryl derivatives substituted at para- and meta-positions (Scheme 1). ${ }^{[16]}$ The use of those substrates is advantageous because steric requirements of para- or meta-substituted benzhydryl derivatives are similar, due to remote position of the substituents from the reaction center. Furthermore, the reactivity of the substrates can easily be adjusted with the choice of the substituents on the benzhydryl rings, enabling characterization of a wide variety of leaving groups by conventional kinetic techniques. For characterization of the poor leaving groups, the LGs in question have been combined with stabilized benzhydrylium ions (good electrofuges), while destabilized benzhydrylium ions (poor electrofuges) have been used for characterization of the good leaving groups (good nucleofuges). 
Originally, the solvolysis rates (at $25^{\circ} \mathrm{C}$ ) in commonly used solvents of substrates that were the combination of 39 differently substituted benzhydryl electrofuges ( $E_{f}$ values in the range of -13 and +6 ) and 14 nucleofuges (in total 101 reference nucleofuge and solvent combinations) were used in optimization procedure according to Equation (1) to obtain the reference $E_{f}$ and also $N_{f}$ and $s_{f}$ parameters.[16] Later, these electrofuges have been employed for further determination of nucleofugalities of series of leaving groups.

Once the electrofugalities of the reference electrofuges have been determined, characterization of a wide diversity of leaving groups has been carried out. The solvolysis rate constants of compounds with investigated LGs were measured using either conductometry ${ }^{[4,5 a, 8,12,17,18]}$ or potentiometric titration at $25^{\circ} \mathrm{C} \cdot{ }^{[19]}$ In a few cases, rate constants were collected from at least three different higher or lower temperatures and were extrapolated to 25 ${ }^{\circ} \mathrm{C}$. For conductometric determination of rate constants, the increase in conductivity during solvolysis was monitored automatically. In order to achieve complete ionization of a liberated acid, either the proton sponge base, lutidine or triethylamine was added at a range of concentrations in which the linear response of conductivity to the increase of the concentration of the liberated acid was observed. For potentiometric measurements, the $\mathrm{pH}$-stat was employed. Typically, a substrate was dissolved in a given solvent and the liberated acid was being continuously titrated with a diluted solution (either 0.008 $\mathrm{M}$ or $0.016 \mathrm{M}$ ) of $\mathrm{NaOH}$ in the same solvent at constant $\mathrm{pH}$ (usually about 7).

In order to obtain the nucleofugality $\left(N_{\mathrm{f}}\right)$ and the slope $\left(s_{f}\right)$ parameters for a variety of leaving groups in

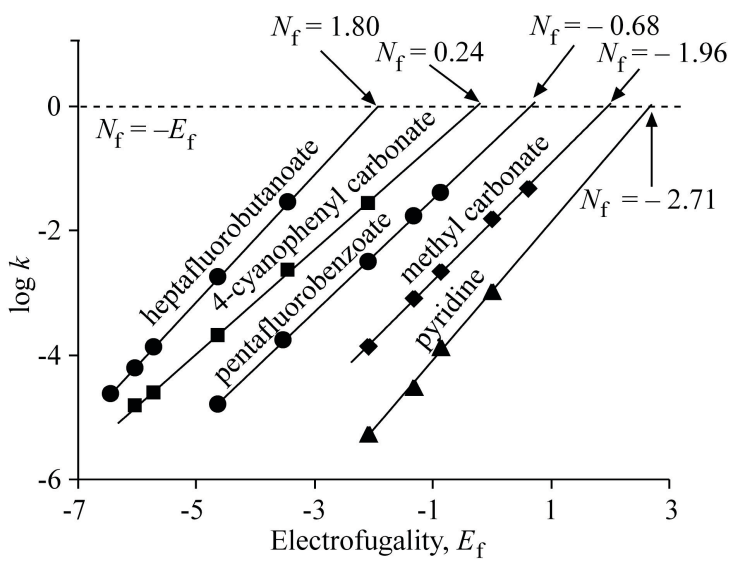

Figure 1. Plots of $\log k\left(25^{\circ} \mathrm{C}\right)$ vs. $E_{\mathrm{f}}$ for solvolyses of substituted benzhydryl heptafluorobutanoate, ${ }^{[16]}$ 4-cyanophenyl carbonate, ${ }^{[4]}$ pentafluorobenzoate, ${ }^{[18]}$ methyl carbonate $^{[16]}$ and pyridine ${ }^{[19 c]}$ in $80 \%$ aq. ethanol $(v / v)$. different solvents and solvent mixtures, the logarithms of first-order rate constants for solvolysis of various benzhydryl derivatives were plotted against the corresponding electrofugalities. Typically, the examined nucleofuge was combined with four or five different electrofuges. The representative plots obtained by applying Equation (1) are presented in Figure 1, in which the correlation lines obtained for $\mathrm{X}, \mathrm{Y}$-substituted benzhydrylLG in $80 \%$ aqueous ethanol are presented. As mentioned above and shown in Figure 1, the negative intercept of the correlation line on the abscissa represents the corresponding nucleofugality $\left(-N_{\mathrm{f}}\right)$, while the slope of the correlation line $s_{\mathrm{f}}$ represents the reaction constant for a given LG in a given solvent. The nucleofuge specific parameters $\left(N_{\mathrm{f}}\right.$ and $\left.s_{\mathrm{f}}\right)$ determined in various solvents are shown in Table 1, in which the nucleofuge-specific parameters of halogens, aliphatic and aromatic carboxylates, aliphatic and aromatic carbonates, as well as phenolates and sulfonates are listed.

Beside neutral substrates, which generate a carbocation and a negatively charged leaving group in the slow heterolytic step, Equation (1) can also be applied for estimating the solvolysis rates of positively charged substrates, such are e.g. pyridinium and sulfonium salts, from which neutral leaving groups are generated. ${ }^{[19]}$ The nucleofuge specific parameters $\left(N_{\mathrm{f}}\right.$ and $\left.s_{\mathrm{f}}\right)$ for some neutral nucleofuges are also included into Table 1 . The main difference in solvolytic behavior between the neutral and charged substrates is that the reactivity of the former increases with solvent polarity, while that of the latter decreases, due to solvation effects in the reactant ground state, as exemplified by entry 37 (Table 1).[19,20]

\section{CALCULATED NUCLEOFUGE- SPECIFIC PARAMETERS}

Beside experimental data used for estimating the nucleofugality parameters of leaving groups, quantum chemical calculations have also been employed.[18,21] The heterolytic transition state structure of various neutral substrates that produce a carbocation and a negatively charged nucleofuge cannot be optimized by standard quantum chemical calculations. Therefore, model reactions, in which the departure of a leaving group occurs in a concerted manner with the neighboring group assistance, have been considered. Further, the calculated barriers obtained for the model reaction have been correlated with the experimental ones obtained in solvolysis of corresponding benzhydryl derivatives. For predicting the nucleofugalities of the series of benzoates, the epoxy ring formation reaction, starting from negatively charged 2-oxyethyl benzoates (Scheme 2a), in which the 
Table 1. Nucleofuge-specific parameters $\left(N_{f}\right.$ and $\left.s_{f}\right)$ for some leaving groups in aqueous binary mixtures

\begin{tabular}{|c|c|c|c|c|c|}
\hline \multirow{2}{*}{ Entry } & \multirow{2}{*}{ Leaving group } & \multicolumn{4}{|c|}{$N_{f} / S_{f}$} \\
\hline & & $80 E 20 W^{(a)}$ & $60 \mathrm{E} 40 \mathrm{~W}^{(\mathrm{a})}$ & 60AN40W(a) & $60 \mathrm{~A} 40 \mathrm{~W}^{(\mathrm{a})}$ \\
\hline 1 & $\mathrm{OTs}^{(\mathrm{b})}$ & $7.45 / 0.80$ & & $7.97 / 0.82$ & \\
\hline 2 & $\mathrm{OMs}^{(\mathrm{b})}$ & $7.49 / 0.84$ & & $7.70 / 0.83$ & \\
\hline 3 & $\mathrm{Br} \mathrm{r}^{(\mathrm{b})}$ & $4.36 / 0.95$ & & $5.23 / 0.99$ & $4.67 / 0.97$ \\
\hline 4 & $\mathrm{Cl}^{(b)}$ & $3.24 / 0.99$ & $4.09 / 0.97$ & $3.84 / 0.96$ & $3.30 / 0.97$ \\
\hline 5 & $F^{(c)}$ & $-1.24 / 0.92$ & & $-1.47 / 0.83$ & $-2.23 / 0.79$ \\
\hline 6 & 4-Nitrophenyl carbonate $\left(4-\mathrm{NO}_{2}-\mathrm{C}_{6} \mathrm{H}_{4} \mathrm{OCO}_{2}\right)^{(\mathrm{d})}$ & $0.31 / 0.80$ & $1.05 / 0.80$ & $0.27 / 0.80$ & $0.12 / 0.81$ \\
\hline 7 & 4-Cyanophenyl carbonate $\left(4-\mathrm{CN}-\mathrm{C}_{6} \mathrm{H}_{4} \mathrm{OCO}\right)^{(\mathrm{d})}$ & $0.24 / 0.84$ & $0.82 / 0.80$ & $0.10 / 0.80$ & $-0.03 / 0.83$ \\
\hline 8 & 4-Chlorophenyl carbonate $\left(4-\mathrm{Cl}-\mathrm{C}_{6} \mathrm{H}_{4} \mathrm{OCO}_{2}\right)^{(\mathrm{d})}$ & $-0.46 / 0.84$ & $0.00 / 0.80$ & $-0.62 / 0.79$ & $-0.80 / 0.84$ \\
\hline 9 & 4-Fluorophenyl carbonate $\left(4-\mathrm{F}-\mathrm{C}_{6} \mathrm{H}_{4} \mathrm{OCO}_{2}\right)^{(\mathrm{d})}$ & $-0.49 / 0.90$ & $-0.08 / 0.84$ & $-0.79 / 0.81$ & $-0.94 / 0.87$ \\
\hline 10 & Phenyl carbonate $\left(\mathrm{PhOCO}_{2}\right)^{(\mathrm{b})}$ & $-0.74 / 0.90$ & $-0.40 / 0.81$ & & $-1.39 / 0.83$ \\
\hline 11 & 4-Methoxyphenyl carbonate $\left(4-\mathrm{MeO}-\mathrm{C}_{6} \mathrm{H}_{4} \mathrm{OCO}_{2}\right)^{(\mathrm{d})}$ & $-0.88 / 0.91$ & $-0.57 / 0.82$ & $-1.24 / 0.81$ & $-1.52 / 0.84$ \\
\hline 12 & 4-Methylphenyl carbonate $\left(4-\mathrm{Me}-\mathrm{C}_{6} \mathrm{H}_{4} \mathrm{OCO}\right)^{(\mathrm{d})}$ & $-0.80 / 0.92$ & $-0.52 / 0.84$ & $-1.26 / 0.80$ & $-1.55 / 0.84$ \\
\hline 13 & Methyl carbonate $\left(\mathrm{MeOCO}_{2}\right)^{(\mathrm{b})}$ & $-1.96 / 0.95$ & $-1.59 / 0.89$ & & $-2.56 / 0.88$ \\
\hline 14 & Ethyl carbonate $\left(\mathrm{EtOCO}_{2}\right)^{(\mathrm{d})}$ & $-2.04 / 0.99$ & $-1.80 / 0.92$ & $-2.54 / 0.90$ & $-2.72 / 0.93$ \\
\hline 15 & Isopropyl carbonate $\left(i \mathrm{PrOCO}_{2}\right)^{(\mathrm{d})}$ & $-2.26 / 1.00$ & $-2.03 / 0.93$ & $-2.94 / 0.88$ & $-2.86 / 0.97$ \\
\hline 16 & Isobutyl carbonate $\left(i \mathrm{BuOCO}_{2}\right)^{(\mathrm{b})}$ & & $-2.04 / 0.89$ & & \\
\hline 17 & Tertbutyl carbonate $\left(t \mathrm{BuOCO}_{2}\right)^{(\mathrm{b})}$ & $-3.12 / 0.96$ & $-2.91 / 0.89$ & $-3.28 / 0.96$ & $-3.62 / 0.94$ \\
\hline 18 & Fluoroacetate $(F A c)^{(e, f)}$ & $-1.72 / 1.00$ & $-1.47 / 0.92$ & $-2.38 / 0.91$ & $-2.40 / 0.94$ \\
\hline 19 & Chloroacetate $(\mathrm{ClAc})^{(\mathrm{e}, \mathrm{f})}$ & $-1.95 / 1.01$ & $-1.75 / 0.93$ & $-2.58 / 0.93$ & $-2.59 / 0.97$ \\
\hline 20 & Bromoacetate $(\mathrm{BrAc})^{(\mathrm{e}, \mathrm{f})}$ & $-1.93 / 1.02$ & $-1.72 / 0.94$ & $-2.60 / 0.93$ & $-2.60 / 0.97$ \\
\hline 21 & Dichloroacetate $(\mathrm{DCIAC})^{(\mathrm{e}, \mathrm{f})}$ & $-0.59 / 0.91$ & $-0.24 / 0.85$ & $-0.87 / 0.85$ & $-1.07 / 0.87$ \\
\hline 22 & Trifluoroacetate (TFAc) $)^{(\mathrm{b}, \mathrm{f})}$ & $1.42 / 0.82$ & $2.11 / 0.82$ & $1.90 / 0.86$ & $1.66 / 0.86$ \\
\hline 23 & Trichloroacetate (TCIAC) ${ }^{(\mathrm{e}, \mathrm{f})}$ & $1.21 / 0.90$ & $1.70 / 0.87$ & $1.49 / 0.89$ & $1.09 / 0.86$ \\
\hline 24 & Heptafluorobutanoate (HFB) $)^{(b, f)}$ & $1.80 / 0.88$ & $2.30 / 0.86$ & $2.16 / 0.89$ & $1.86 / 0.88$ \\
\hline 25 & Formate (Form) ${ }^{(e, f)}$ & $-2.13 / 1.04$ & $-1.87 / 0.95$ & $-2.67 / 0.93$ & $-2.70 / 0.98$ \\
\hline 26 & Acetate $(A c)^{(b, e, g)}$ & $-3.61 / 1.12$ & $-3.63 / 1.00$ & $-4.18 / 1.08$ & $-4.05 / 1.17$ \\
\hline 27 & 2-Methylpropanoate (Isobutyrate) (e,f,g,h) & $-3.97 / 1.15$ & $-4.25 / 1.02$ & $-4.92 / 1.01$ & $-4.71 / 1.10$ \\
\hline 28 & 2,2-Dimethylpropanoate (Pivalate) (e,f,g,h) & $-4.29 / 1.17$ & $-4.54 / 1.03$ & $-5.32 / 1.02$ & \\
\hline 29 & Pentafluorobenzoate (PFB) $)^{(i, j)}$ & $-0.68 / 0.90$ & & $-1.12 / 0.87$ & $-1.15 / 0.92$ \\
\hline 30 & Trifluorobenzoate (TFB) ${ }^{(i, j)}$ & $-1.75 / 0.98$ & & $-2.05 / 0.95$ & $-2.30 / 0.97$ \\
\hline 31 & 3,5-Dinitrobenzoate (DNB) $)^{(b)}$ & $-1.43 / 0.98$ & & $-2.06 / 0.97$ & $-2.20 / 0.90$ \\
\hline 32 & 4-Nitrobenzoate (PNB) ${ }^{(b)}$ & $-2.78 / 0.95$ & & $-3.30 / 0.91$ & $-2.79 / 1.11$ \\
\hline 33 & 2-Nitrobenzoate ${ }^{(j)}$ & & & $-2.30 / 0.94$ & $-2.53 / 0.98$ \\
\hline 34 & Benzoate $(\mathrm{BzO})^{(\mathrm{b})}$ & & & $-3.92 / 1.02$ & $-3.89 / 1.15$ \\
\hline 35 & 2,4-Dinitrophenolate (DNP) $)^{(k)}$ & $0.22 / 1.03$ & & & $-0.14 / 0.98$ \\
\hline 36 & Pentafluorophenolate (PFP)(I) & $-0.97 / 1.29$ & & $-0.63 / 1.34$ & $-1.12 / 1.21$ \\
\hline 37 & Dimethylsulfide $\left(\mathrm{Me}_{2} \mathrm{~S}\right)^{(\mathrm{b})}$ & $1.96 / 0.86$ & $1.83 / 0.86$ & & \\
\hline 38 & Tetrahydrothiophene $(\mathrm{THT})^{(\mathrm{m})}$ & $2.20 / 0.86$ & & & \\
\hline 39 & 4-Chloropyridine (4-CIPy) ${ }^{(n)}$ & $-1.37 / 1.14$ & & & \\
\hline 40 & Pyridine $(P y)^{(n)}$ & $-2.71 / 1.10$ & & & \\
\hline 41 & 4-Methylpyridine (4-MePy) ${ }^{(n)}$ & $-3.48 / 1.12$ & & & \\
\hline
\end{tabular}

(a) Binary solvents are expressed as volume fractions at $25^{\circ} \mathrm{C}$ : $\mathrm{E}=$ ethanol, $\mathrm{AN}=$ acetonitrile, $\mathrm{A}=$ acetone, and $\mathrm{W}=$ water

(b) Data are taken from Ref. [16].

(c) Data are taken from Ref. [24].

(d) Data are taken from Ref. [4].

(e) Data are taken from Ref. [8]

(f) Data are taken from Ref. [17f].

(g) $s_{\mathrm{f}}$ values were estimated from the $s_{\mathrm{f}} / \log k$ correlations of dianisylmethyl carboxylates (Ref. [8]).

(h) $s_{\mathrm{f}}$ values were estimated from the $s_{\mathrm{f}} / \log k$ correlations of dianisylmethyl carboxylates (Ref. [17f]).

Data are taken from Ref. [18].

(j) Data are taken from Ref. [17e]

(k) Data are taken from Ref. [12].

(I) Data are taken from Ref. [5a].

(m) Data are taken from Ref. [19b]

(n) Data are taken from Ref. [19c] 
(a)

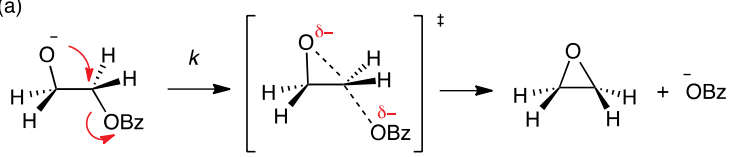

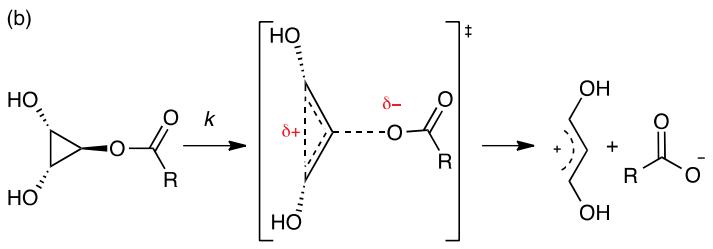

Scheme 2.

intramolecular backside $n$-electron attack of the negatively charged oxygen is a driving force for the carbon-benzoate bond cleavage, has been used. ${ }^{[18]}$ For determination of the nucleofugalities of aliphatic carboxylates, the model presented in Scheme $2 \mathrm{~b}$ was found to be a suitable model reaction. In that model reaction anchimerically assisted heterolytic dissociation of cis-2,3-dihydroxycyclopropyl trans-carboxylates occurs. [21] The reaction barriers presented in Scheme 2 had been calculated by using both B3LYP and M06-2X DFT methods in the presence of the IEFPCM solvation model representing water as a solvent, and then, they were correlated with the corresponding experimental barriers obtained for solvolysis of the series of 4,4'dimethoxybenzhydryl benzoates and aliphatic carboxylates, respectively, $\left(\mathrm{X}=\mathrm{Y}=4-\mathrm{OCH}_{3}\right.$ in Scheme 1$)$ in various solvents. Both model reactions were justified by very good correlation plots $(r=0.994-0.999 ;$ MAE $=0.09-0.30 \mathrm{kcal}$ $\mathrm{mol}^{-1}$ with slopes of $\left.0.89-1.00\right) .{ }^{[17 e, f, 18,21]}$ The plot obtained by correlating $\Delta H^{\text {fmodel }}$ for heterolysis of the model benzoates with $\Delta G^{\ddagger}$ for solvolysis of corresponding 4,4'-dimethoxybenzhydryl benzoates in $80 \%$ aq. ethanol is given in Figure 2a. ${ }^{[18]}$

The $\Delta G^{\ddagger}$ vs. $\Delta G^{\ddagger}$ model correlation plot for aliphatic carboxylates in which $\Delta G^{\ddagger \text { model }}$ were calculated at the M062X/AUG-cc-pVTZ level in the presence of the IEFPCM solvation model and the experimental $\Delta G^{\ddagger} \mathrm{s}$ obtained in 80 $\%$ aq. ethanol, is shown in Figure 2b. ${ }^{[21]}$

Quantum chemical modeling has further been employed to obtain energy barriers for the anchimerically assisted heterolysis of numerous of both 2-oxyethyl benzoates and 2,3-dihydroxycyclopropyl carboxylates. Using the calculated barriers of the model reactions and relationships derived from the $\Delta G^{\ddagger}\left(4,4^{\prime}\right.$-dimethoxybenzhydryl-LG) vs. $\Delta G^{\ddagger \text { model }}$ (model reaction) correlations, free energies of activation have been estimated for solvolysis of various 4,4'-dimethoxybenzhydryl aromatic and aliphatic carboxylate derivatives in aqueous ethanol, acetone and acetonitrile mixtures. ${ }^{[17 e, f, 18,21]}$ (a)

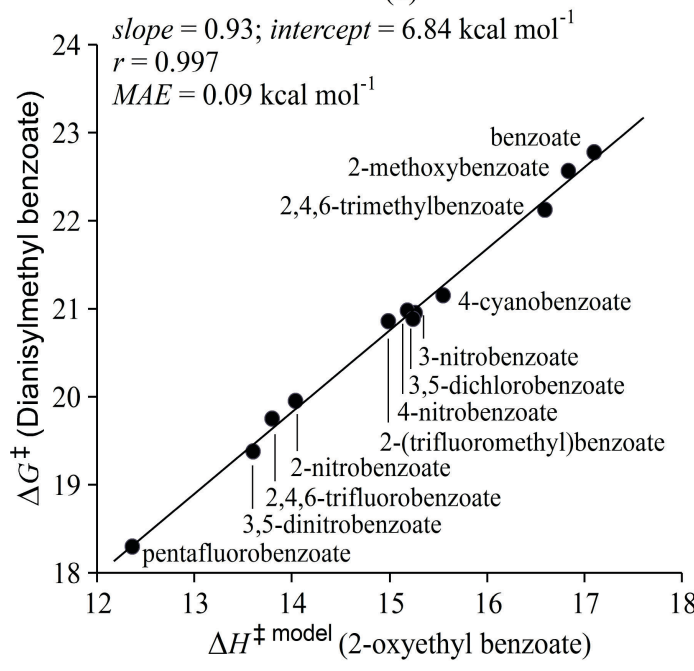

(b)

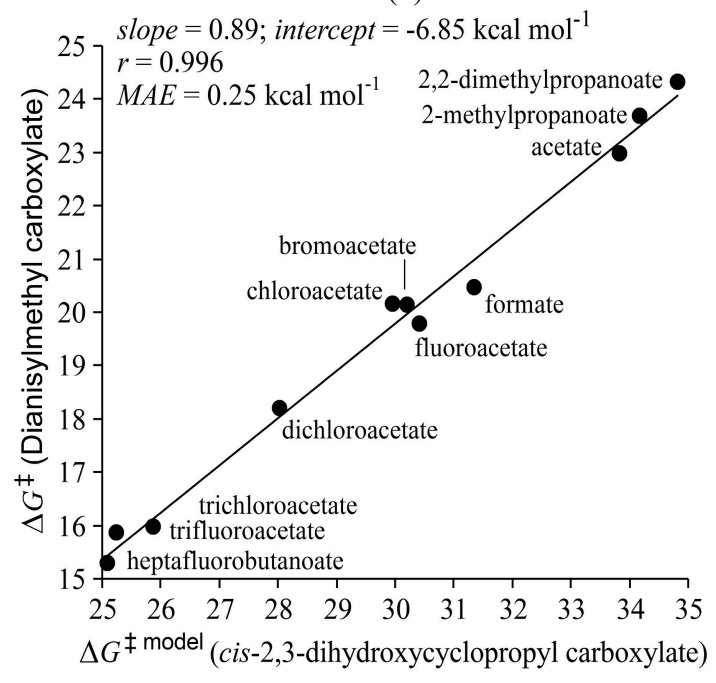

Figure 2. Correlation of experimental activation free energies ( $\mathrm{kcal} \mathrm{mol}^{-1}$ ) for solvolyses of (a) dianisylmethyl benzoates in $80 \%$ aq. ethanol at $25{ }^{\circ} \mathrm{C}$ vs. enthalpies of activation for heterolyses of 2-oxyethyl benzoates calculated at the B3LYP/6-311+G(2d,p) level of theory in the presence of the IEFPCM solvation model (solvent = water) ${ }^{[18]}$ and (b) dianisylmethyl carboxylates in $80 \%$ aq. ethanol vs. free energies of activation (in $\mathrm{kcal} \mathrm{mol}^{-1}$ ) for heterolysis of cis-2,3-dihydroxycyclopropyl carboxylates calculated at the M06-2X/AUG-cc-pVTZ level of theory in the presence of the IEFPCM solvation model (solvent = water). [21]

The estimated values of $s_{f}$ for different carboxylate leaving groups were based both on the similarity in the structure of the LGs (benzoates) and on the good $s_{f} v s$. log $k\left(4,4^{\prime}-\mathrm{di}\right.$ methoxybenzhydryl-LG) correlations (aliphatic carboxylates). 
Table 2. Calculated nucleofugalities $\left(N_{\mathrm{f}}\right.$ calc) and the corresponding estimated reaction constants $\left(s_{\mathrm{f}}\right.$ estim) for some leaving groups in aqueous binary mixtures

\begin{tabular}{|c|c|c|c|c|c|}
\hline \multirow{2}{*}{ Entry } & \multirow{2}{*}{ Leaving group } & \multicolumn{4}{|c|}{$N_{f}^{\text {calc }} / S_{f}^{\text {estim }}$} \\
\hline & & $80 E 20 W^{(a)}$ & $60 \mathrm{E} 40 \mathrm{~W}^{(\mathrm{a})}$ & 60AN40W(a) & $60 \mathrm{~A} 40 \mathrm{~W}^{(\mathrm{a})}$ \\
\hline 1 & 2,2-Dimethylpropanoate ${ }^{(c, d)}$ (Pivalate) & & & & $-5.01 / 1.12$ \\
\hline 2 & Propanoate $e^{(b, c, d)}$ & $-3.63 / 1.12$ & $-3.57 / 1.00$ & $-4.43 / 0.99$ & $-4.33 / 1.08$ \\
\hline 3 & Butanoate $^{(b, c, d)}$ & $-3.83 / 1.14$ & $-3.84 / 1.01$ & $-4.73 / 1.00$ & $-4.60 / 1.09$ \\
\hline 4 & Phenylacetate ${ }^{(b, c, d)}$ & $-3.22 / 1.10$ & $-3.10 / 0.98$ & $-3.87 / 0.98$ & $-3.86 / 1.06$ \\
\hline 5 & Propenoate $\mathrm{b}^{(\mathrm{b}, \mathrm{c}, \mathrm{d})}$ & $-3.11 / 1.09$ & $-2.95 / 0.98$ & $-3.71 / 0.98$ & $-3.75 / 1.05$ \\
\hline 6 & Propynoate $\mathrm{e}^{(\mathrm{b}, \mathrm{c}, \mathrm{d})}$ & $-1.13 / 0.98$ & $-0.64 / 0.91$ & $-1.16 / 0.92$ & $-1.47 / 0.95$ \\
\hline 7 & Difluoroacetate ${ }^{(b, c, d)}$ & $-0.20 / 0.93$ & $0.39 / 0.88$ & $-0.04 / 0.89$ & $-0.41 / 0.91$ \\
\hline 8 & Dibromoacetate $\mathrm{e}^{(\mathrm{b}, \mathrm{c}, \mathrm{d})}$ & $-0.04 / 0.92$ & $0.58 / 0.87$ & $0.16 / 0.89$ & $-0.23 / 0.90$ \\
\hline 9 & Tribromoacetate $\mathrm{b}^{(\mathrm{b}, \mathrm{c}, \mathrm{d})}$ & $0.82 / 0.88$ & $1.49 / 0.85$ & $1.14 / 0.87$ & $0.74 / 0.87$ \\
\hline 10 & Pentafluoropropanoate ${ }^{(b, c, d)}$ & $1.68 / 0.85$ & $2.39 / 0.83$ & $2.10 / 0.85$ & $1.70 / 0.84$ \\
\hline 11 & Pentachloropropanoate ${ }^{(b, c, d)}$ & $0.84 / 0.88$ & $1.52 / 0.85$ & $1.17 / 0.87$ & $0.76 / 0.87$ \\
\hline 12 & Pentabromopropanoate ${ }^{(b, c, d)}$ & $0.61 / 0.89$ & $1.27 / 0.86$ & $0.90 / 0.88$ & $0.50 / 0.88$ \\
\hline 13 & Heptachlorobutanoate ${ }^{(b, c, d)}$ & $0.84 / 0.88$ & $1.51 / 0.85$ & $1.16 / 0.87$ & $0.75 / 0.87$ \\
\hline 14 & 3,3,3-Trifluoropropanoate $\mathrm{b}^{(b, c, d)}$ & $-1.70 / 1.01$ & $-1.28 / 0.93$ & $-1.87 / 0.93$ & $-2.11 / 0.98$ \\
\hline 15 & Hexafluoroisobutanoate ${ }^{(b, c, d)}$ & $-0.56 / 0.95$ & $0.00 / 0.89$ & $-0.46 / 0.90$ & $-0.82 / 0.92$ \\
\hline 16 & Nonafluorotrimethylacetate ${ }^{(b, c, d)}$ & $2.24 / 0.83$ & $2.95 / 0.82$ & $2.70 / 0.84$ & $2.32 / 0.82$ \\
\hline 17 & Cyanoacetate $e^{(b, c, d)}$ & $-1.16 / 0.98$ & $-0.67 / 0.91$ & $-1.18 / 0.92$ & $-1.49 / 0.95$ \\
\hline 18 & Dicyanoacetate $e^{(b, c, d)}$ & $2.10 / 0.83$ & $2.79 / 0.82$ & $2.54 / 0.84$ & $2.17 / 0.82$ \\
\hline 19 & Tricyanoacetate $\mathrm{e}^{(\mathrm{b}, \mathrm{c}, \mathrm{d})}$ & $6.21 / 0.70$ & $6.67 / 0.74$ & $6.50 / 0.78$ & $6.59 / 0.71$ \\
\hline 20 & Nitroacetate ${ }^{(b, c, d)}$ & $-0.27 / 0.93$ & $0.33 / 0.88$ & $-0.11 / 0.90$ & $-0.49 / 0.91$ \\
\hline 21 & Dinitroacetate $\mathrm{b}^{(\mathrm{b}, \mathrm{c}, \mathrm{d})}$ & $3.12 / 0.80$ & $3.83 / 0.80$ & $3.60 / 0.83$ & $3.31 / 0.79$ \\
\hline 22 & Trinitroacetate ${ }^{(b, c, d)}$ & $7.49 / 0.67$ & $7.79 / 0.72$ & $7.65 / 0.76$ & $7.97 / 0.68$ \\
\hline 23 & 2-Cyanopropenoate ${ }^{(b, c, d)}$ & $-0.90 / 0.96$ & $-0.37 / 0.90$ & $-0.87 / 0.91$ & $-1.19 / 0.94$ \\
\hline 24 & 2-Hydroxyethanoate ${ }^{(b, c, d)}$ & $-2.17 / 1.03$ & $-1.83 / 0.94$ & $-2.45 / 0.95$ & $-2.64 / 1.00$ \\
\hline 25 & 2-Hydroxypropanoate ${ }^{(b, c, d)}$ & $-2.39 / 1.04$ & $-2.07 / 0.95$ & $-2.74 / 0.95$ & $-2.89 / 1.01$ \\
\hline 26 & 2,3-Dihydroxypropanoate $\mathrm{e}^{(\mathrm{b}, \mathrm{c}, \mathrm{d})}$ & $-2.01 / 1.02$ & $-1.64 / 0.94$ & $-2.26 / 0.94$ & $-2.47 / 0.99$ \\
\hline 27 & Oxoethanoate ${ }^{(b, c, d)}$ & $-0.28 / 0.93$ & $0.31 / 0.88$ & $-0.13 / 0.90$ & $-0.50 / 0.91$ \\
\hline 28 & 2-Oxopropanoate ${ }^{(b, c, d)}$ & $-1.47 / 0.99$ & $-1.01 / 0.92$ & $-1.57 / 0.93$ & $-1.83 / 0.97$ \\
\hline 29 & 3-Oxopropanoate ${ }^{(b, c, d)}$ & $-3.18 / 1.09$ & $-3.03 / 0.98$ & $-3.79 / 0.98$ & $-3.82 / 1.05$ \\
\hline 30 & 2-Oxobutanoate $e^{(b, c, d)}$ & $-1.69 / 1.00$ & $-1.26 / 0.93$ & $-1.85 / 0.93$ & $-2.08 / 0.98$ \\
\hline 31 & 3-Oxobutanoate $\mathrm{e}^{(\mathrm{b}, \mathrm{c}, \mathrm{d})}$ & $-3.13 / 1.09$ & $-2.97 / 0.98$ & $-3.73 / 0.98$ & $-3.77 / 1.05$ \\
\hline 32 & Oxalate, 1. dissociation ${ }^{(b, c, d)}$ & $-0.66 / 0.95$ & $-0.10 / 0.89$ & $-0.58 / 0.91$ & $-0.93 / 0.93$ \\
\hline 33 & Oxalate, 2. dissociation ${ }^{(b, c, d)}$ & $-3.80 / 1.14$ & $-3.81 / 1.01$ & $-4.69 / 1.00$ & $-4.57 / 1.09$ \\
\hline 34 & Malonate, 1. dissociation $(b, c, d)$ & $-2.79 / 1.07$ & $-2.59 / 0.96$ & $-3.28 / 0.97$ & $-3.39 / 1.03$ \\
\hline 35 & Malonate, 2. dissociation ${ }^{(b, c, d)}$ & $-4.59 / 1.19$ & $-4.79 / 1.04$ & $-5.78 / 1.03$ & $-5.47 / 1.14$ \\
\hline 36 & Benzoate ${ }^{(e, f)}$ & $-4.09 / 0.95$ & & & \\
\hline 37 & 2-Nitrobenzoate $e^{(e, f)}$ & $-1.89 / 0.95$ & & & \\
\hline 38 & 3-Nitrobenzoate ${ }^{(e, f, g)}$ & $-2.77 / 0.95$ & & $-3.19 / 0.91$ & $-2.88 / 1.11$ \\
\hline 39 & 2,4-Dinitrobenzoate (e,f,g) & $-0.55 / 0.98$ & & $-1.30 / 0.98$ & $-1.10 / 0.90$ \\
\hline 40 & 2,6-Dinitrobenzoate (e,f,g) & $-0.60 / 0.98$ & & $-1.35 / 0.98$ & $-1.16 / 0.90$ \\
\hline 41 & 3,4-Dinitrobenzoate $\mathrm{e}^{(\mathrm{e}, \mathrm{f}, \mathrm{g})}$ & $-1.63 / 0.98$ & & $-2.40 / 0.98$ & $-2.34 / 0.90$ \\
\hline 42 & 3,4,5-Trinitrobenzoate ${ }^{(e, f, g)}$ & $-0.75 / 0.98$ & & $-1.48 / 1.00$ & $-1.24 / 0.97$ \\
\hline 43 & 2,4,6-Trinitrobenzoate ${ }^{(e, f, g)}$ & $0.53 / 0.98$ & & $-0.20 / 1.00$ & $0.12 / 0.97$ \\
\hline 44 & 2-Cyanobenzoate ${ }^{(e, f, g)}$ & $-2.63 / 0.95$ & & $-3.24 / 1.00$ & $-3.12 / 0.98$ \\
\hline 45 & 3-Cyanobenzoate ${ }^{(e, f, g)}$ & $-2.99 / 0.95$ & & $-3.70 / 0.98$ & $-3.08 / 1.11$ \\
\hline 46 & 4-Cyanobenzoate ${ }^{(e, f, g)}$ & $-2.97 / 0.95$ & & $-3.39 / 0.91$ & $-3.07 / 1.11$ \\
\hline 47 & 2,4-Dicyanobenzoate ${ }^{(e, f, g)}$ & $-1.56 / 0.98$ & & $-2.33 / 0.98$ & $-2.26 / 0.90$ \\
\hline 48 & 2,6-Dicyanobenzoate ${ }^{(e, f, g)}$ & $-0.49 / 0.98$ & & $-1.24 / 0.98$ & $-1.03 / 0.90$ \\
\hline 49 & 3,5-Dicyanobenzoate ${ }^{(e, f, g)}$ & $-1.88 / 0.98$ & & $-2.66 / 0.98$ & $-2.63 / 0.90$ \\
\hline 50 & 3,4-Dicyanobenzoate ${ }^{(e, f, g)}$ & $-1.91 / 0.98$ & & $-2.69 / 0.98$ & $-2.66 / 0.90$ \\
\hline 51 & 3,4,5-Tricyanobenzoate $e^{(e, f, g)}$ & $-1.03 / 0.98$ & & $-1.76 / 1.00$ & $-1.54 / 0.97$ \\
\hline 52 & 2,4,6-Tricyanobenzoate ${ }^{(e, f, g)}$ & $0.47 / 0.98$ & & $-0.25 / 1.00$ & $0.06 / 0.97$ \\
\hline 53 & Pentacyanobenzoate $\mathrm{e}^{(\mathrm{e}, \mathrm{fg})}$ & $2.02 / 0.90$ & & $1.24 / 0.91$ & $1.62 / 0.92$ \\
\hline 53 & 3-Formylbenzoate ${ }^{(e, f, g)}$ & $-3.21 / 0.95$ & & $-3.92 / 0.98$ & $-3.28 / 1.11$ \\
\hline 54 & 4-Formylbenzoate ${ }^{(e, f, g)}$ & $-3.19 / 0.95$ & & $-3.90 / 0.98$ & $-3.26 / 1.11$ \\
\hline 55 & 3,5-Diformylbenzoate ${ }^{(e, f, g)}$ & $-2.55 / 0.98$ & & $-3.35 / 0.98$ & $-3.40 / 0.90$ \\
\hline 56 & 2-(Trifluoromethyl)benzoate $\mathrm{e}^{(\mathrm{e}, \mathrm{f}, \mathrm{g})}$ & $-1.57 / 0.95$ & & $-2.89 / 0.94$ & $-3.06 / 0.98$ \\
\hline 57 & 3-(Trifluoromethyl)benzoate $e^{(\mathrm{e}, \mathrm{f}, \mathrm{g})}$ & $-3.28 / 0.95$ & & $-3.99 / 0.98$ & $-3.34 / 1.11$ \\
\hline 58 & 4-(Trifluoromethyl)benzoate ${ }^{(e, f, g)}$ & $-3.29 / 0.95$ & & $-3.99 / 0.98$ & $-3.35 / 1.11$ \\
\hline
\end{tabular}

(The table is continued on the next page.) 
Table 2. (Continued from the previous page)

\begin{tabular}{|c|c|c|c|c|c|}
\hline \multirow{2}{*}{ Entry } & \multirow{2}{*}{ Leaving group } & \multicolumn{4}{|c|}{$N_{\mathrm{f}}^{\text {calc }} / \mathrm{S}_{\mathrm{f}}^{\text {estim }}$} \\
\hline & & $80 E 20 W^{(a)}$ & $60 E 40 W^{(a)}$ & 60AN40W(a) & $60 \mathrm{~A} 40 \mathrm{~W}^{(\mathrm{a})}$ \\
\hline 59 & 2,4-Bis(trifluoromethyl)benzoate ${ }^{(e, f, g)}$ & $-1.66 / 0.98$ & & $-2.43 / 0.98$ & $-2.37 / 0.90$ \\
\hline 60 & 2,6-Bis(trifluoromethyl)benzoate ${ }^{(e, f, g)}$ & $-1.37 / 0.98$ & & $-2.14 / 0.98$ & $-2.05 / 0.90$ \\
\hline 61 & 3,5-Bis(trifluoromethyl)benzoate ${ }^{(e, f, g)}$ & $-2.35 / 0.98$ & & $-2.88 / 0.90$ & $-3.17 / 0.90$ \\
\hline 62 & 3,4-Bis(trifluoromethyl)benzoate ${ }^{(e, f, g)}$ & $-2.40 / 0.98$ & & $-3.19 / 0.98$ & $-3.22 / 0.90$ \\
\hline 63 & $3,4,5$-Tris(trifluoromethyl)benzoate ${ }^{(e, f, g)}$ & $-1.69 / 0.98$ & & $-2.41 / 1.00$ & $-2.23 / 0.97$ \\
\hline 64 & $2,4,6$-Tris(trifluoromethyl)benzoate ${ }^{(e, f, g)}$ & $-0.52 / 0.98$ & & $-1.25 / 1.00$ & $-1.00 / 0.97$ \\
\hline 65 & Penta(trifluoromethyl)benzoate $e^{(e, f, g)}$ & $0.55 / 0.90$ & & $-0.24 / 0.91$ & $0.11 / 0.92$ \\
\hline 66 & 2-Chlorobenzoate ${ }^{(e, f, g)}$ & $-2.74 / 0.95$ & & $-3.35 / 1.01$ & $-3.24 / 0.98$ \\
\hline 67 & 3-Chlorobenzoate ${ }^{(e, f, g)}$ & $-3.41 / 0.95$ & & $-4.12 / 0.98$ & $-3.46 / 1.11$ \\
\hline 68 & 4-Chlorobenzoate ${ }^{(e, f, g)}$ & $-3.67 / 0.95$ & & $-4.37 / 0.98$ & $-3.69 / 1.11$ \\
\hline 69 & 2,4-Dichlorobenzoate ${ }^{(e, f, g)}$ & $-2.44 / 0.98$ & & $-3.23 / 0.98$ & $-3.27 / 0.90$ \\
\hline 70 & 2,6-Dichlorobenzoate ${ }^{(e, f, g)}$ & $-1.47 / 0.98$ & & $-2.24 / 0.98$ & $-2.16 / 0.90$ \\
\hline 71 & 3,5-Dichlorobenzoate ${ }^{(e, f, g)}$ & $-2.67 / 0.98$ & & $-3.21 / 0.90$ & $-3.53 / 0.90$ \\
\hline 72 & 3,4-Dichlorobenzoate ${ }^{(e, f, g)}$ & $-2.99 / 0.98$ & & $-3.79 / 0.98$ & $-3.90 / 0.90$ \\
\hline 73 & $3,4,5$-Trichlorobenzoate $e^{(e, f, g)}$ & $-2.47 / 0.98$ & & $-3.20 / 1.00$ & $-3.07 / 0.97$ \\
\hline 74 & 2,4,6-Trichlorobenzoate ${ }^{(e, f, g)}$ & $-1.13 / 0.98$ & & $-1.86 / 1.00$ & $-1.64 / 0.97$ \\
\hline 75 & Pentachlorobenzoate ${ }^{(e, f, g)}$ & $-0.57 / 0.90$ & & $-1.37 / 0.91$ & $-1.05 / 0.92$ \\
\hline 76 & 3-Fluorobenzoate ${ }^{(e, f, g)}$ & $-3.52 / 0.95$ & & $-4.23 / 0.98$ & $-3.56 / 1.11$ \\
\hline 77 & 4-Fluorobenzoate ${ }^{(e, f, g)}$ & $-3.87 / 0.95$ & & $-4.57 / 0.98$ & $-3.88 / 1.11$ \\
\hline 78 & 2,4-Difluorobenzoate ${ }^{(e, f, g)}$ & $-3.11 / 0.98$ & & $-3.92 / 0.98$ & $-4.05 / 0.90$ \\
\hline 79 & 2,6-Difluorobenzoate ${ }^{(e, f, g)}$ & $-1.67 / 0.98$ & & $-2.44 / 0.98$ & $-2.39 / 0.90$ \\
\hline 80 & 3,5-Difluorobenzoate ${ }^{(e, f, g)}$ & $-2.82 / 0.98$ & & $-3.62 / 0.98$ & $-3.71 / 0.90$ \\
\hline 81 & 3,4-Difluorobenzoate ${ }^{(e, f, g)}$ & $-3.19 / 0.98$ & & $-4.00 / 0.98$ & $-4.13 / 0.90$ \\
\hline 82 & $3,4,5$-Trifluorobenzoate $e^{(e, f, g)}$ & $-2.64 / 0.98$ & & $-3.37 / 1.00$ & $-3.25 / 0.97$ \\
\hline 83 & 2-Phenylbenzoate ${ }^{(e, f, g)}$ & $-3.71 / 0.95$ & & $-4.28 / 1.01$ & $-4.23 / 0.98$ \\
\hline 84 & 3-Phenylbenzoate ${ }^{(e, f, g)}$ & $-4.01 / 0.95$ & & $-4.71 / 0.98$ & $-4.00 / 1.11$ \\
\hline 85 & 4-Phenylbenzoate ${ }^{(e, f, g)}$ & $-4.16 / 0.95$ & & $-4.86 / 0.98$ & $-4.14 / 1.11$ \\
\hline 86 & 3,5-Diphenylbenzoate ${ }^{(e, f, g)}$ & $-3.82 / 0.98$ & & $-4.64 / 0.98$ & $-4.85 / 0.90$ \\
\hline 87 & 2-Methoxybenzoate $\mathrm{e}^{(\mathrm{f}, \mathrm{g})}$ & $-3.89 / 0.95$ & & $-4.16 / 0.94$ & $-4.41 / 0.98$ \\
\hline 88 & 3-Methoxybenzoate ${ }^{(e, f, g)}$ & $-4.10 / 0.95$ & & $-4.80 / 0.98$ & $-4.08 / 1.11$ \\
\hline 89 & 4-Methoxybenzoate ${ }^{(\mathrm{e}, \mathrm{f}, \mathrm{g})}$ & $-4.66 / 0.95$ & & $-5.36 / 0.98$ & $-4.59 / 1.11$ \\
\hline 90 & 2,4-Dimethoxybenzoate $e^{(e, f, g)}$ & $-4.68 / 0.98$ & & $-5.52 / 0.98$ & $-5.84 / 0.90$ \\
\hline 91 & 2,6-Dimethoxybenzoate ${ }^{(e, f, g)}$ & $-3.44 / 0.98$ & & $-4.25 / 0.98$ & $-4.41 / 0.90$ \\
\hline 92 & 3,5-Dimethoxybenzoate $\mathrm{e}^{(\mathrm{e}, \mathrm{f}, \mathrm{g})}$ & $-3.93 / 0.98$ & & $-4.75 / 0.98$ & $-4.98 / 0.90$ \\
\hline 93 & 3,4-Dimethoxybenzoate ${ }^{(e, f, g)}$ & $-4.48 / 0.98$ & & $-5.32 / 0.98$ & $-5.62 / 0.90$ \\
\hline 94 & $3,4,5$-Trimethoxybenzoate $e^{(e, f, g)}$ & $-4.05 / 0.98$ & & $-4.78 / 1.00$ & $-4.75 / 0.97$ \\
\hline 95 & $2,4,6$-Trimethoxybenzoate ${ }^{(e, f, g)}$ & $-3.79 / 0.98$ & & $-4.51 / 1.00$ & $-4.47 / 0.97$ \\
\hline 96 & 2-Methylbenzoate ${ }^{(e, f, g)}$ & $-4.37 / 0.95$ & & $-4.92 / 1.01$ & $-4.90 / 0.98$ \\
\hline 97 & 3-Methylbenzoate $e^{(e, f, g)}$ & $-4.32 / 0.95$ & & $-5.02 / 0.98$ & $-4.28 / 1.11$ \\
\hline 98 & 4-Methylbenzoate $e^{(e, f, g)}$ & $-4.41 / 0.95$ & & $-5.11 / 0.98$ & $-4.36 / 1.11$ \\
\hline 99 & 2,4-Dimethylbenzoate ${ }^{(e, f, g)}$ & $-4.57 / 0.98$ & & $-5.41 / 0.98$ & $-5.72 / 0.90$ \\
\hline 100 & 2,6-Dimethylbenzoate ${ }^{(e, f, g)}$ & $-3.35 / 0.98$ & & $-4.16 / 0.98$ & $-4.31 / 0.90$ \\
\hline 101 & 3,5-Dimethylbenzoate ${ }^{(e, f, g)}$ & $-4.29 / 0.98$ & & $-5.12 / 0.98$ & $-5.40 / 0.90$ \\
\hline 102 & 3,4-Dimethylbenzoate ${ }^{(e, f, g)}$ & $-4.43 / 0.98$ & & $-5.26 / 0.98$ & $-5.55 / 0.90$ \\
\hline 103 & 3,4,5-Trimethylbenzoate ${ }^{(e, f, g)}$ & $-4.58 / 0.98$ & & $-5.31 / 1.00$ & $-5.32 / 0.97$ \\
\hline 104 & 2,4,6-Trimethylbenzoate ${ }^{(\mathrm{e}, \mathrm{f}, \mathrm{g})}$ & $-3.61 / 0.98$ & & $-3.96 / 0.95$ & $-4.28 / 0.97$ \\
\hline 105 & Pentamethylbenzoate ${ }^{(e, f, g)}$ & $-4.17 / 0.90$ & & $-5.01 / 0.91$ & $-4.77 / 0.92$ \\
\hline 106 & 3-Aminobenzoate ${ }^{(e, f, g)}$ & $-4.40 / 0.95$ & & $-5.10 / 0.98$ & $-4.36 / 1.11$ \\
\hline 107 & 4-Aminobenzoate ${ }^{(e, f, g)}$ & $-5.21 / 0.95$ & & $-5.89 / 0.98$ & $-5.08 / 1.11$ \\
\hline 108 & 3,5-Diaminobenzoate ${ }^{(e, f, g)}$ & $-4.49 / 0.98$ & & $-5.33 / 0.98$ & $-5.63 / 0.90$ \\
\hline 109 & 3,4,5-Triaminobenzoate $e^{(e, f, g)}$ & $-5.18 / 0.98$ & & $-5.91 / 1.00$ & $-5.95 / 0.90$ \\
\hline
\end{tabular}

(a) Binary solvents are expressed as volume fractions at $25^{\circ} \mathrm{C}$ : $\mathrm{E}=$ ethanol, $\mathrm{AN}=$ acetonitrile, $\mathrm{A}=$ acetone, and $\mathrm{W}=$ water.

(b) Data are taken from Ref. [21].

(c) Data are taken from Ref. [17f].

(d) $s_{\mathrm{f}}$ parameters estimated from the correlation of $s_{\mathrm{f}}$ versus $\log k\left(25^{\circ} \mathrm{C}\right)$ for solvolysis of dianisylmethyl carboxylates in an appropriate solvent (Refs. [8] and [17f]). $N_{\mathrm{f}}$ parameters were calculated from the $\log k^{\text {calc }}$ and appropriate $s_{\mathrm{f}}^{\text {estim }}$ by using equation $\log k=s_{\mathrm{f}}\left(E_{\mathrm{f}}+N_{\mathrm{f}}\right)$ (Refs. [21] and [17f]). $E_{\mathrm{f}}$ value for the dianisylmethyl electrofuge is 0.00 . (Ref. [16])

(e) Data are taken from Ref. [18].

(f) $N_{\mathrm{f}}$ parameters were calculated from $k^{\text {calc }}$ and related $s_{\mathrm{f}}$ using equation $\log k=s_{\mathrm{f}}\left(E_{\mathrm{f}}+N_{\mathrm{f}}\right)$ (Ref. [18]). $E_{\mathrm{f}}$ value for dianisylmethyl electrofuge is 0.00 (Ref. [16]). Applied experimental $s_{f}$ values used for calculating $N_{f}$ values (Refs. [18],[17e] and [16]).

(g) Data are taken from Ref. [17e]. 
Once the rate constants of 4,4'-dimethoxybenzhydryl-LG $\left(E_{f}=0\right)$ and the reaction constants $\left(s_{f}\right)$ have been estimated, the corresponding nucleofugality parameters for various combination of carboxylate leaving groups and solvents have been derived by applying Equation (1).[17e,f,18,21] The estimated nucleofuge-specific parameters are presented in Table 2 . Since the calculated nucleofugalities $\left(N_{\mathrm{f}}^{\mathrm{calc}}\right)$ deviate negligibly from the experimentally determined $N_{\mathrm{f}}$ values ( $M A E=0.16-0.23$ for various $\mathrm{LG} /$ solvent combinations), ${ }^{[17, f, 18,21]}$ they can be taken as reliable values in a further application along with experimentally determined ones.

\section{APPLICATION OF THE NUCLEOFUGALITY PARAMETERS}

The above presented LFER model (equation 1) and the nucleofugality/electrofugality scales can be used for estimating the relative reactivities of leaving groups, as well as for predicting the absolute solvolysis rate of a given substrate in a given solvent.

By comparing the $N_{\mathrm{f}}$ values, the relative reactivities of the leaving groups can be estimated in most of the cases. Having in mind that the unit of $N_{f}$ corresponds to one order of magnitude difference in reactivity, it can, for example, from Table 1 be determined that halogen substituents in an aliphatic moiety of carboxylates alter the reactivities of aliphatic carboxylates up to six orders of magnitude (entries 18-26 in Table 1), ${ }^{[8,16]}$ as well as that tosylate (entry 1, Table $1)^{[16]}$ is more reactive leaving group than 4-nitrobenzoate (entry 32$)^{[16]}$ for about 10 orders of magnitude. By analyzing the variation of the nucleofugalities, the impact of a solvent on solvolytic reactivity of various substrates can also be considered. Thus, e.g. phenyl carbonates (entry 10, Table 1), ${ }^{[16]}$ solvolyze faster in $60 \%$ aq. ethanol than the corresponding dichloroacetates in $80 \%$ aq. ethanol (entry 21, Table 1$),{ }^{[8]}$ even though the latter is a better leaving group. It is also obvious that the effect of a solvent is much more pronounced with neutral substrates than with charged substrates that produce neutral leaving groups. For instance, chloroacetates, bromoacetates and also other carboxylates solvolyze for one order of magnitude faster in $80 \%$ aq. ethanol than in $80 \%$ aq. acetonitrile (the difference of $N_{\mathrm{f}}$ values is about one unit; for bromoacetate $N_{f}=-1.93$ in $80 \%$ aq. EtOH and $N_{f}=-2.92$ in $80 \%$ aq. acetonitrile), ${ }^{[8,17 f]}$ while the difference of the nucleofugality for the pyridine leaving group (produced from pyridinium ions) is only 0.1 units ( $N_{f}=-2.71$ in $80 \%$ aq. EtOH and $N_{\mathrm{f}}=-2.64$ in $80 \%$ aq. acetonitrile). ${ }^{[19 c, d]}$

The other nucleofuge-specific parameter, the reaction constant $s_{\mathrm{f}}$, similarly as Hammett-Brown $\rho^{+}$constant, indicates the amount of the positive charge generated on the reaction center in the heterolytic transition state. By

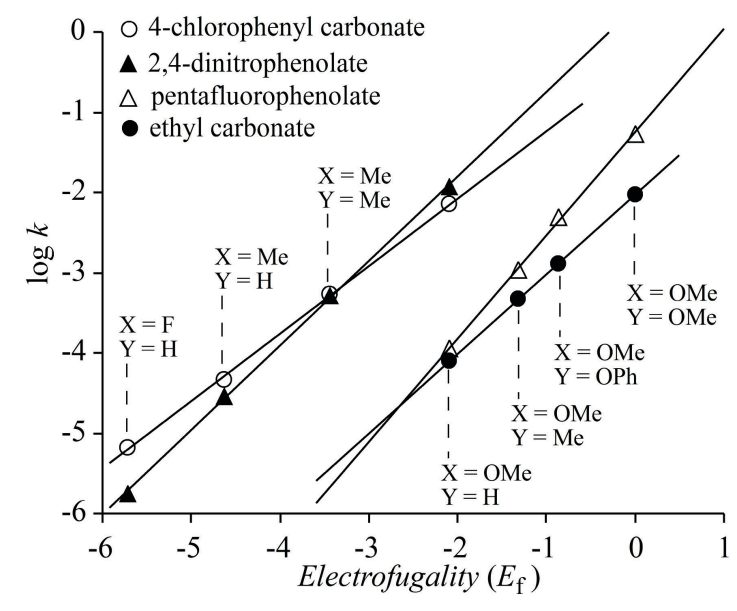

Figure 3. Plots of $\log k\left(25^{\circ} \mathrm{C}\right)$ vs. $E_{f}$ for solvolyses of substituted benzhydryl aryl and alkyl carbonates, ${ }^{[4]}$ 2,4dinitrophenolates (DNPh) ${ }^{[12]}$ and pentafluorophenolates $(\mathrm{PFPh})^{[5]}$ in $80 \%$ aq. ethanol $(\mathrm{v} / \mathrm{v})$.

studying the magnitude and the variation of the reaction constants, fine electronic and solvation effects that determine the solvolytic reactivity and the structure of the transition state have been established, and described in details. ${ }^{[5 b, 17 c, 22]}$

Due to small variation of $s_{f}$ parameter (ranges from 0.77 to 1.36 ), it only slightly influences the reaction rate (equation 1), so comparison of the sole $N_{f}$ parameters generally indicates the relative reactivities of the leaving groups correctly. However, when $N_{f}$ values of two compared LGs are close in magnitude, while the corresponding $s_{f}$ parameters differ substantially in the above mentioned range, the intersection of the corresponding $\log k v s$. $E_{\mathrm{f}}$ plots may occur in the experimentally accessible range of reactivity, indicating the inversion in relative reactivity of the leaving groups. Some examples are shown in Figure 3, in which the log $k v s$. $E_{f}$ correlation lines in $80 \%$ aq. ethanol are presented for 2,4-dinitrophenolates $\left(N_{\mathrm{f}}=0.22, s_{\mathrm{f}}=\right.$ 1.03), ${ }^{[12]}$ 4-chlorophenyl carbonates $\left(N_{f}=-0.46, s_{f}=0.84\right),{ }^{[4]}$ pentafluorophenolates $\left(N_{f}=-0.97, s_{f}=1.29\right),{ }^{[5]}$ and ethyl carbonates $\left(N_{f}=-2.04, s_{f}=0.99\right) .{ }^{[4]}$ Because of steeper log $k v s$. $E_{\mathrm{f}}$ correlation plots for phenolates than for carbonates, lines for phenolates intersect the lines for carbonates. ${ }^{[4]}$ Pentafluorophenolates constituted from electrofuges whose $E_{\mathrm{f}}<-2.5$ solvolyze slower than corresponding ethyl carbonates, but those constituted from more stable electrofuges solvolyze faster. Similarly, the intersection of the correlation lines for 2,4-dinitrophenolates and 4-chlorophenyl carbonates occurs at $E_{\mathrm{f}} \approx-3.5$, indicating that 4,4'dimethylbenzhydryl 2,4-dinitrophenolate and 4-chlorophenyl carbonate $\left(E_{\mathrm{f}}=-3.47\right)$ solvolyze with similar reaction rate, however, 2,4-dinitrophenolates with less reactive 
electrofuges solvolyze slower and those with more reactive electrofuges faster than corresponding 4-chlorophenyl

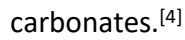

Accordingly, by using the above LFER model it has been shown that the relative reactivities of leaving groups greatly depend on the electrofuge moiety (electrofugality) of the substrate. This phenomenon cannot be established if the relative reactivities of the leaving groups are studied using substrates that are the combination of a single electrofuge and different nucleofuges. ${ }^{[4,5]}$

In everyday laboratory practice it is sometimes necessary to know the approximate value of the absolute reaction rate of solvolysis of a given substrate in a given solvent, in order to decide if the half-life of the substrate is long enough to allow it to be handled in the solvent of choice. Therefore, one of the applications of the nucleofugality/electrofugality scales may be prediction of the rate constant according to Equation (1) for solvolysis of a substrate constituted from any electrofuge-nucleofuge combination in a given solvent. The electrofugalities for structurally diverse electrofuges were determined using literature data and the reference nucleofuges. ${ }^{[23]}$ Since the above LFER model has been developed employing benzhydryl derivatives, the solvolysis rates of benzhydryl derivatives, as well as of substrates with aromatic electrofuges can be predicted quite reliably. Although obtained $E_{f}$ values of aliphatic electrofuges deviate in various solvents (up to one order of magnitude) due to differential solvation of the electrofuge moieties, the estimated half-lives are still sufficiently accurate to be taken as a reliable indicator of substrate reactivities in various solvents at $25^{\circ} \mathrm{C}$. ${ }^{[23]}$

It is illustrative to compare the predicted reactivities and reaction half-lives for some substrates in $80 \%$ aq. ethanol which are shown in Table 3. The adamantyl electrofuge is one of the weakest electrofuge $\left(E_{\mathrm{f}}=-11.7 \pm 0.7\right.$ in $80 \%$ aq. ethanol), ${ }^{[23]}$ so the substrate that is the combination of the adamantyl electrofuge and a moderate or weak nucleofuge is stable for a reasonably period of time in variety of solvents at ambient temperature. Adamantyl 3methoxybenzoate, which is the combination of both a very week electrofuge and a very week nucleofuge (entry 88 , in Table 2) is a particularly stable compound, whose half-live of reaction in various solvents is about 6 million years. On the other hand, adamantyl tosylate and mesylate are relatively unstable compounds in various solvents at ambient temperature. They solvolyze with rates accessible for standard kinetic measurements.

If it is desirable to have a stable substrate constituted of a moderate electrofuge, such are those with e.g. the allylic moiety (example given with 2-cyclohexenyl electrofuge, $E_{f}=-6.33 \pm 0.41$, Table 3), ${ }^{[23]}$ the nucleofuge moiety

Table 3. Estimated solvolysis rates in $80 \%$ aq. ethanol and corresponding half-lives for some selected substrates.

\begin{tabular}{|c|c|c|c|c|}
\hline Substrate & Leaving group & $\log k^{\text {estim (f) }}$ & $\mathrm{kestim}^{\mathrm{es}} / \mathrm{s}^{-1}$ & $t_{1 / 2}$ estim \\
\hline \multirow[b]{4}{*}{$E_{\mathrm{f}}=-11.1 \pm 0.7^{(\mathrm{a})}$} & tosylate ${ }^{(c)}$ & -2.9 & $1.2 \times 10^{-3}$ & $10 \mathrm{~min}$ \\
\hline & bromide ${ }^{(c)}$ & -6.4 & $4.0 \times 10^{-7}$ & 21 days \\
\hline & trifluoroacetate ${ }^{(c)}$ & -7.9 & $1.2 \times 10^{-8}$ & 1.9 years \\
\hline & 3-methoxybenzoate ${ }^{(d)}$ & -14.4 & $3.6 \times 10^{-15}$ & 6 million years \\
\hline \multirow{5}{*}{$E_{f}=-6.33$} & bromide $e^{(c)}$ & -1.9 & $1.4 \times 10^{-2}$ & $52 \mathrm{~s}$ \\
\hline & trifluoroacetate ${ }^{(c)}$ & -4.0 & $9.3 \times 10^{-5}$ & $2 \mathrm{~h}$ \\
\hline & methyl carbonate $e^{(\mathrm{c})}$ & -7.9 & $1.3 \times 10^{-8}$ & 1.7 years \\
\hline & 3-methoxybenzoate ${ }^{(\mathrm{d})}$ & -9.9 & $1.2 \times 10^{-10}$ & 180 years \\
\hline & trifluoroacetate ${ }^{(c)}$ & 1.2 & $1.4 \times 10^{1}$ & $48 \mathrm{~ms}$ \\
\hline \multirow[b]{2}{*}{$E_{\mathrm{f}}=0.00^{(\mathrm{b}}$} & methyl carbonate ${ }^{(c)}$ & -1.9 & $1.4 \times 10^{-2}$ & $51 \mathrm{~s}$ \\
\hline & 4-methylpyridine $e^{(e)}$ & -3.9 & $1.3 \times 10^{-4}$ & $1.5 \mathrm{~h}$ \\
\hline
\end{tabular}

\footnotetext{
(a) Electrofuge parameters are taken from Ref. [23].

(b) Electrofuge parameter is taken from Ref. [16].

(c) Nucleofuge parameters are taken from Ref. [16].

(d) Nucleofuge parameters are taken from Ref. [18].

(e) Nucleofuge parameters are taken from Ref. [19c].

(f) Estimated by using Equation ( 1 ) and corresponding $E_{f}, N_{f}$ and $s_{f}$ parameters.
} 
should be chosen carefully. While allylic electrofuge in combination with bromide (or chloride) solvolyzes in aqueous alcohol in a few seconds, and in the combination with a moderate nucleofuge reacts in a short period of time, the allylic electrofuge combined with a poor leaving group, such is for example 3-methoxybenzoate, is a stable compound with $t_{1 / 2}$ in $80 \%$ aq. ethanol for about 180 years.

On contrary, compounds formed from very good electrofuges, such is, for example, the 4,4'-dimethoxybenzhydryl electrofuge $\left(E_{f}=0\right),{ }^{[16]}$ are unstable even in combination with very poor nucleofuges (Table 3 ).

Advantageously, the above LFER model based on Equation (1) provides information about the stability of the<smiles></smiles>

$E_{f}=5.6$<smiles></smiles>

$E_{f}=-12.9$
Scheme 3.

substrates in wide range of reactivities. Thus, half-lives as well as first order solvolysis rate constants of substrates assembled from the so far most reactive electrofuge $\left(E_{\mathrm{f}}=\right.$ 5.6 , Scheme 3 ) and the most reactive nucleofuge (entry 1 , Table 1) and that of the least reactive both electrofuge $\left(E_{f}\right.$ $=-12.9$, Scheme 3) and nucleofuge (entry 107, Table 2) differ for about 28 orders of magnitude $\left(t_{1 / 2} \approx 10^{-11} \mathrm{~s} v \mathrm{~s}\right.$. $10^{17} \mathrm{~s}, 10^{9}$ years; $k \approx 10^{10} \mathrm{~s}^{-1}$ vs. $10^{-18} \mathrm{~s}^{-1}$ ), so the former reaction is diffusion controlled process.

Acknowledgments. The authors gratefully acknowledge financial support of this research by the Croatian Science Foundation (under the project number IP-1021).

\section{REFERENCES}

[1] (a) M. B. Smith, J. March, March's Advanced Organic Chemistry: Reactions, Mechanisms and Structure, 6th ed., A John Wiley \& Sons, Inc.; Hoboken, New Jersey, 2007. (b) T. H. Lowry, K. S. Richardson, Mechanism and Theory in Organic Chemistry, 3rd ed., Harper \& Row, New York, 1987.

[2] (a) F. A. Carey, R. J. Sundberg, Advanced Organic Chemistry, Part A. 3 rd. Ed. Plenum Press, New York, 1990. (b) S. Borčić, O. Kronja, K. Humski, Croat. Chem. Acta 1994, 67(2), 171. (c) C. J. Collins, N. S. Brown, Isotope Effects in Chemical Reactions, Eds. Van Nostrand Reinhold Company, New York, 1971. (d) L. Melender, W. H. Saunders, Jr., Reaction Rates of Isotopic Molecules, Wiley, New York, 1980. (e) Y Okamoto, H. C. Brown, J. Org. Chem. 1957, 22, 485. (f) A. H. Fainberg, S. Winstein, J. Am. Chem. Soc. 1957, 79, 1602. (g) T. W. Bentley, P. v R. Schleyer, Adv. Phys. Org. Chem. 1977, 14, 1. (h) D. N. Kevill, Advances in Quantitative Structure-Property Relationships, Vol. 1 (Ed.: M. Charton), JAl Press, Greenwich, CT, 1996, pp. 81-115. (i) Y. Tsuno, M. Fujio, Adv. Phys. Org. Chem. 1999, 32, 267. (j) D. A. Semenow, J. D. Roberts, J. Chem. Educ. 1956, 33(1), 2. (k) B. K. Carpenter, Determination of Organic Reaction Mechanisms, John Wiley, New York, 1984.

[3] R. A. Marcus, J. Phys. Chem. 1968, 72, 891.

[4] M. Matić, M. Katić, B. Denegri, O. Kronja, J. Org. Chem. 2017, 82, 7820.

[5] (a) M. Matić, N. Bebek, B. Denegri, O. Kronja, Croat. Chem. Acta 2016, 89, 355. (b) B. Denegri, M. Matić, O. Kronja, Synthesis 2017, 49, 3422.

[6] (a) J. E. Leffler, Science 1953, 117, 340. (b) J. E. Leffler, E. Grunwald, Rates and Equilibria of Organic Reactions, Wiley: New York, 1963. (c) G. S. Hammond, J. Am. Chem. Soc. 1955, 77, 334.

[7] M. J. S. Dewar, R. C. Dougherty, The PMO Theory of Organic Chemistry, Plenum, New York, 1975.

[8] M. Matić, B. Denegri, O. Kronja, Eur. J. Org. Chem. 2014, 1477.

[9] B. Denegri, M. Matić, O. Kronja, ChemistrySelect 2016, 1, 5250.

[10] T. W. Bentley, K. Roberts, J. Org. Chem. 1985, 50, 4821.

[11] (a) W. L. Jolly, Modern Inorganic Chemistry, McGraw-Hill, 1984, p. 177. (b) E. P. Serjeant, B. Dempsey, IUPAC Chemical Data Series No 23, Pergamon Press, New York, 1979.

[12] M. Matić, B. Denegri, O. Kronja, Eur. J. Org. Chem. 2010, 6019.

[13] (a) CRC Handbook of Chemistry and Physics, 87th ed., CRC Press; 2006-2007. (b) B. G. Tehan, E. J. Lloyd, M. G. Wong, W. R. Pitt, J. G. Montana, D. T. Manallack, E. Gancia, Quant. Struct.-Act. Relat., 2002, 21(5), 457.

[14] D. S. Noyce, J. A. Virgilio, J. Org. Chem. 1972, 37, 2643.

[15] (a) H. Mayr, T. Bug, M. F. Gotta, N. Hering, B. Irrgang, B. Janker, B. Kempf, R. Loos, A. R. Ofial, G. Remennikov, H. Schimmel, J. Am. Chem. Soc. 2001, 123, 9500. (b) H. Mayr, B. Kempf, A. R. Ofial, Acc. Chem. Res. 2003, 36, 66. (c) H. Mayr, A. R. Ofial, Pure Appl. Chem. 2005, 77, 1807. (d) H. Mayr, A. R. Ofial, J. Phys. Org. Chem. 2008, 21, 584.

[16] N. Streidl, B. Denegri, O. Kronja, H. Mayr, Acc. Chem. Res. 2010, 43, 1537.

[17] (a) B. Denegri, A. Streiter, S. Jurić, A. R. Ofial, O. Kronja, H. Mayr, Chem. - Eur. J., 2006, 12, 1648. (b) B. Denegri, O. Kronja, J. Org. Chem. 2007, 72, 8427. (c) B. Denegri, O. Kronja, J. Phys. Org. Chem. 2009, 22, 495. (d) B. Denegri, O. Kronja, J. Org. Chem. 2009, 74, 5927. (e) M. Matić, B. Denegri, O. Kronja, Croat. 
Chem. Acta. 2012, 85, 585. (f) M. Matić, B. Denegri, O. Kronja, Croat. Chem. Acta. 2014, 87, 375.

[18] M. Matić, B. Denegri, O. Kronja, J. Org. Chem. 2012, $77,8986$.

[19] (a) S. Jurić, B. Denegri, O. Kronja, J. Org. Chem. 2010, 75, 3851. (b) S. Jurić, B. Denegri, O. Kronja, J. Phys. Org. Chem. 2012, 25, 147. (c) S. Jurić, O. Kronja, J. Phys. Org. Chem. 2015, 28, 314. (d) S. Jurić, T. Portolan, O. Kronja, Croat. Chem. Acta 2016, 89, 65.

[20] (a) D. N. Kevill, S. W. Anderson, J. Am. Chem. Soc. 1986, 108, 1579. (b) D. N. Kevill, W. A. Kamil, S. W.
Anderson, Tetrahedron Lett. 1982, 23, 4635. (c) D. N. Kevill, N. H. J. Ismail, M. J. D'Souza, J. Org. Chem. 1994, 59, 6303. (d) D. N. Kevill, S. W. Anderson, N. H. J. Ismail, J. Org. Chem. 1996, 61, 7256.

[21] B. Denegri, M. Matić, O. Kronja, Org. Biomol. Chem. 2014, 12, 5698.

[22] M. Matić, S. Jurić, B. Denegri, O. Kronja, Int. J. Mol. Sci. 2012, 13, 2012.

[23] B. Denegri, A. R. Ofial, S. Jurić, A. Streiter, O. Kronja, H. Mayr, Chem. - Eur. J. 2006, 12, 1657.

[24] C. Nolte, J. Ammer, H. Mayr, J. Org. Chem. 2012, 77, 3325. 